\title{
Two-Dimensional $\mathrm{CH}_{3} \mathrm{NH}_{3} \mathrm{PbI}_{3}$ Perovskite: Synthesis and Optoelectronic Application
}

Jingying Liu ${ }^{\dagger, \|}$, Yunzhou Xue ${ }^{\ddagger, \dagger, \|}$, Ziyu Wang ${ }^{\dagger, \|}$, Zai-Quan $\mathrm{Xu}^{\dagger}$, Changxi Zheng ${ }^{\S}$, Bent Weber ${ }^{\perp}$, Jingchao Song ${ }^{\dagger}$, Yusheng Wang $^{\ddagger}$, Yuerui Lü, Yupeng Zhang*, ${ }^{\dagger}$, and Qiaoliang Bao*,‡,†

${ }^{\dagger}$ Department of Materials Science and Engineering, Monash University, Wellington Road, Clayton, Victoria 3800, Australia.

${ }^{\ddagger}$ Institute of Functional Nano and Soft Materials (FUNSOM), Jiangsu Key Laboratory for Carbon-Based Functional Materials and Devices, and Collaborative Innovation Center of Suzhou Nano Science and Technology, Soochow University, Suzhou 215123, P. R. China.

$\S^{\S}$ Department of Civil Engineering, Monash University, Clayton 3800, Victoria, Australia.

${ }^{\perp}$ School of Physics, Monash University, Monash 3800, Victoria, Australia.

${ }^{\#}$ College of Engineering and Computer Science, Australian National University, Australia

"These authors contributed equally to this work.

*Address correspondence to qlbao@suda.edu.cn (Q. Bao), yupeng.zhang@monash.edu (Y. Zhang)

KEYWORDS: two-dimensional material, hybrid organic-inorganic perovskite, optoelectronics, photodetector 


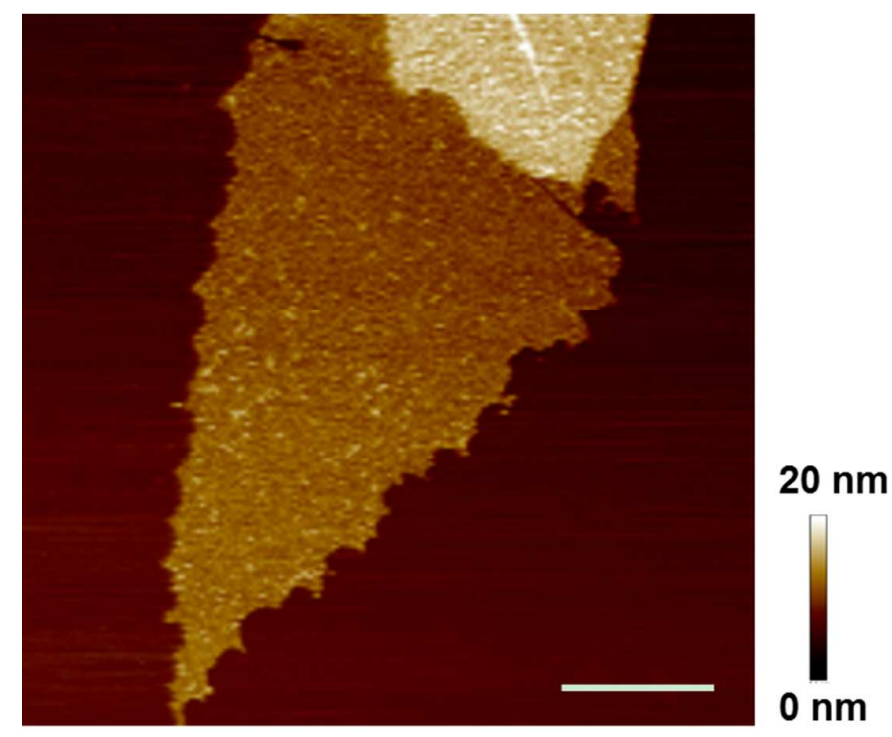

Figure S1. AFM topography image of 2D perovskite nanosheet produced by acombined low temperature solution process and CVD method. Scale bar: $2 \mu \mathrm{m}$.

The low temperature growth process always lasts for 30 mins to 1 hour. An important issue should to be noted is that the $\mathrm{PbI}_{2}$ is also unstable in $\mathrm{H}_{2} \mathrm{O}$. During the slow growth process with low temperature, there is a competitive relationship between the $\mathrm{PbI}_{2}$ crystal growth and re-dissolution, which will affect the crystal quality inevitably. The corresponding AFM image (Figure S1) for perovskite nanosheets reveals the re-dissolution clearly during the solution process, that is, the crystal exhibited the jagged edges. 

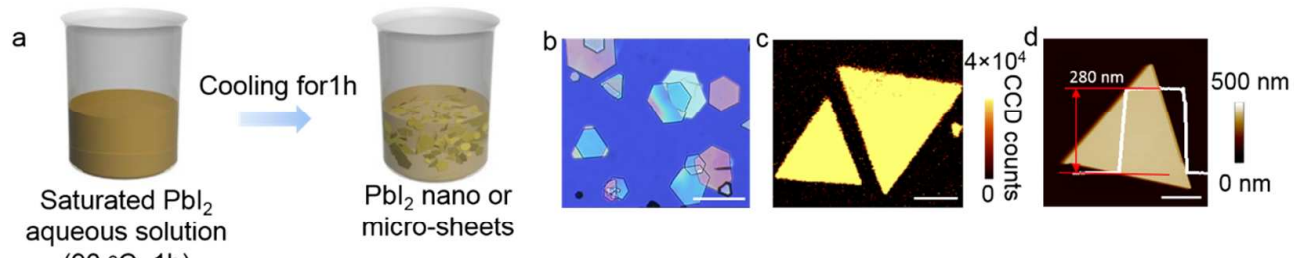
$\left(90^{\circ} \mathrm{C}, 1 \mathrm{~h}\right)$

e
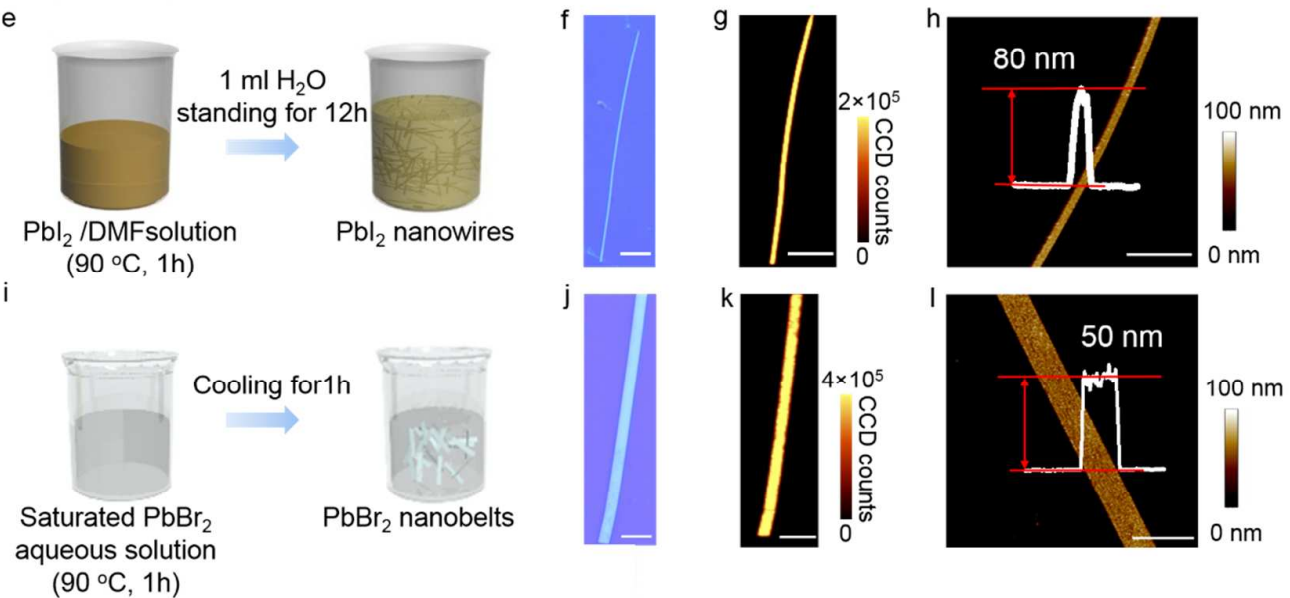

Figure S2. (a) Schematic illustration of solution process to fabricate thick $\mathrm{PbI}_{2}$ nano or microsheets (thickness: 100-300 nm) (b) Optical image for perovskite microsheets. Scale bar: $5 \mu \mathrm{m}$. (c) PL mapping images for perovskite microsheets. Scale bar: $5 \mu \mathrm{m}$. (d) AFM images for perovskite microsheets. Scale bar: $5 \mu \mathrm{m}$. (e) Schematic illustration of solution process to fabricate $\mathrm{PbI}_{2}$ nanowires. (f) Optical image for perovskite nanowires. Scale bar: $3 \mu \mathrm{m}$. (g) PL mapping images for perovskite nanowires. Scale bar: $3 \mu \mathrm{m}$. (h) AFM images for perovskite nanowires. Scale bar: 3 $\mu \mathrm{m}$. (i) Schematic illustration of solution process to fabricate $\mathrm{PbBr}_{2}$ nanobelts. (j) Optical image for perovskite nanobelts. Scale bar: $3 \mu \mathrm{m}$. (k) PL mapping images for perovskite nanobelts. Scale bar: $3 \mu \mathrm{m}$. (1) AFM images for perovskite nanobelts. Scale bar: $3 \mu \mathrm{m}$.

Compared with CVD method to synthesize the $\mathrm{PbI}_{2}$ crystals, the morphology of $\mathrm{PbI}_{2}$ crystals could be more controlled by our solution process. The $\mathrm{PbI}_{2}$ and subsequent $\mathrm{CH}_{3} \mathrm{NH}_{3} \mathrm{PbI}_{3}$ nanowires, nanobelts, and microsheets with different diameters and thicknesses could be obtained, as shown in Figure S2.

$\mathrm{PbI}_{2}$ microsheets: Owing to the different solubility of $\mathrm{PbI}_{2}$ in water at different temperatures, we developed a new crystallization method to prepare $\mathrm{PbI}_{2}$ microsheets. 
By inversing the growth temperature, the resulting $\mathrm{PbI}_{2}$ microsheets with different diameters and thicknesses could be produced (Figure S2a-d).

$\mathrm{PbI}_{2}$ nanowires: When dissolving $0.5 \mathrm{~g} \mathrm{PbI}_{2}$ powder in $\mathrm{N}, \mathrm{N}$-dimethylmethanamide (DMF) solution at $90{ }^{\circ} \mathrm{C}$ for 1 hour, followed by the 12 hours standing time with deionized water added into the $\mathrm{PbI}_{2} / \mathrm{DMF}$ solution, the $\mathrm{PbI}_{2}$ nanowires will precipitate out (Figure S2e-h).

$\mathrm{PbBr}_{2}$ nanobelts: Owing to the different solubility of $\mathrm{PbBr}_{2}$ in water at different temperatures, by inversing the growth temperature, the resulting $\mathrm{PbBr}_{2}$ nanobelts with different diameters and thicknesses could be produced (Figure S2i-1).

Similarly, the $\mathrm{CH}_{3} \mathrm{NH}_{3} \mathrm{PbI}_{3}$ perovskites in different morphology can be formed through intercalating the $\mathrm{CH}_{3} \mathrm{NH}_{3} \mathrm{I}$ molecules into the interval sites of $\mathrm{PbI}_{6}$ octahedrons layers by using CVD method. 


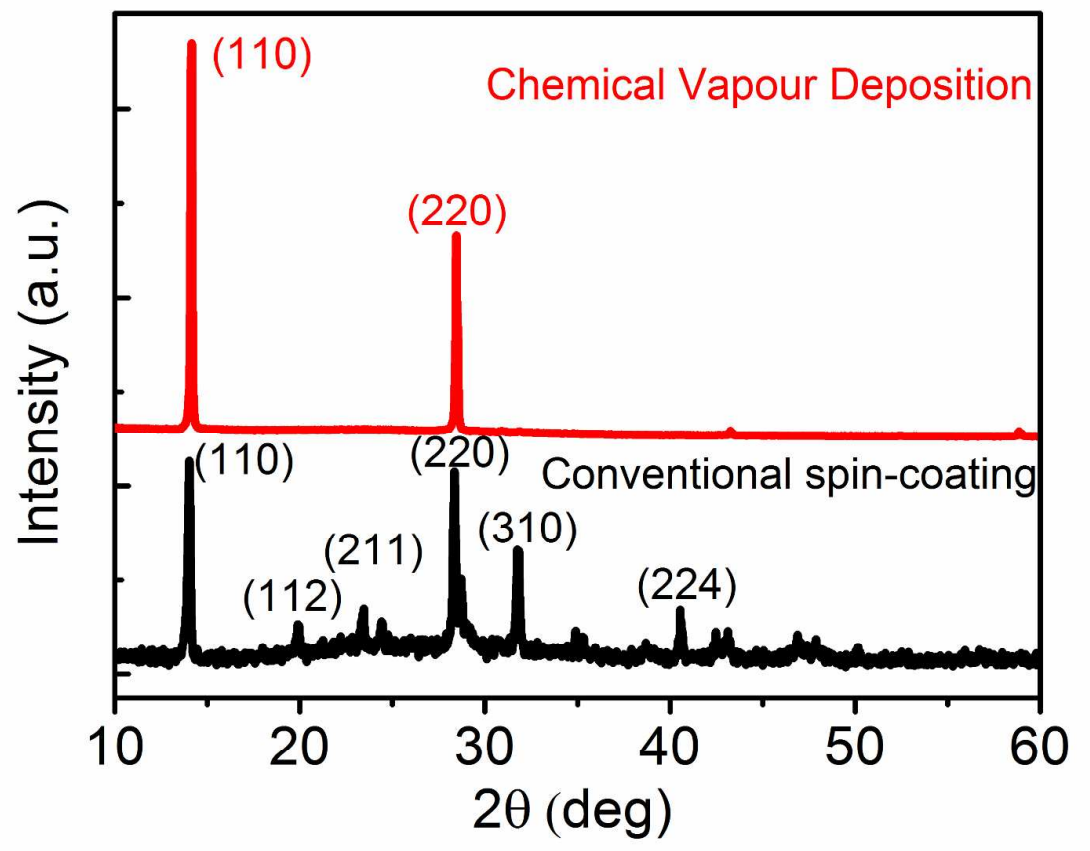

Figure $\mathrm{S} 3 \mathrm{XRD}$ patterns of $\mathrm{CH}_{3} \mathrm{NH}_{3} \mathrm{PbI}_{3}$ perovskite produced by different methods. Red curve, perovskite nanosheets produced by a combined solution process and vapour phase conversion method. Black curve, $\mathrm{CH}_{3} \mathrm{NH}_{3} \mathrm{PbI}_{3}$ film produced by a conventional two step spin-coating method.

XRD patterns are obtained from different samples produced by CVD process (2D nanosheets) and conventional spin-coating (polycrystalline thin film). The XRD patterns of perovskite nanosheets does not reveal the presence of other orientations:

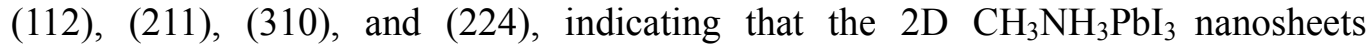
obtained by our method are highly crystallized and have preferred orientation on substrate. 

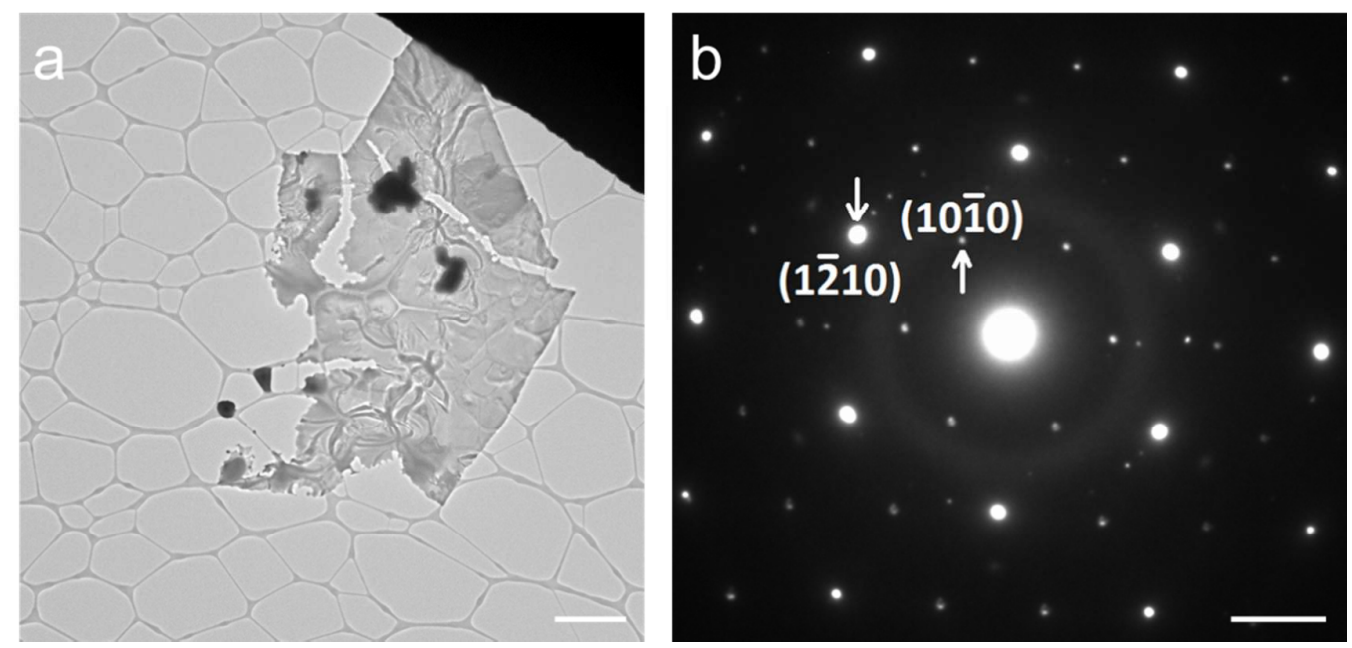

Figure S4 (a) Low magnification TEM image of a 2D PbI 2 nanosheet. Scale bar: $2 \mu \mathrm{m}$. (b) Selected-area electron diffraction pattern of $\mathrm{PbI}_{2}$ nanosheet along the [0001] zone axis. Scale bar: $21 / \mathrm{nm}$.

Figure S4a and b show the low magnification TEM image and the selected-area electron diffraction pattern taken from the as-grown $\mathrm{PbI}_{2}$ nanosheet, indicating a highly crystalline $\mathrm{PbI}_{2}$ with six-fold symmetric diffraction patterns. 


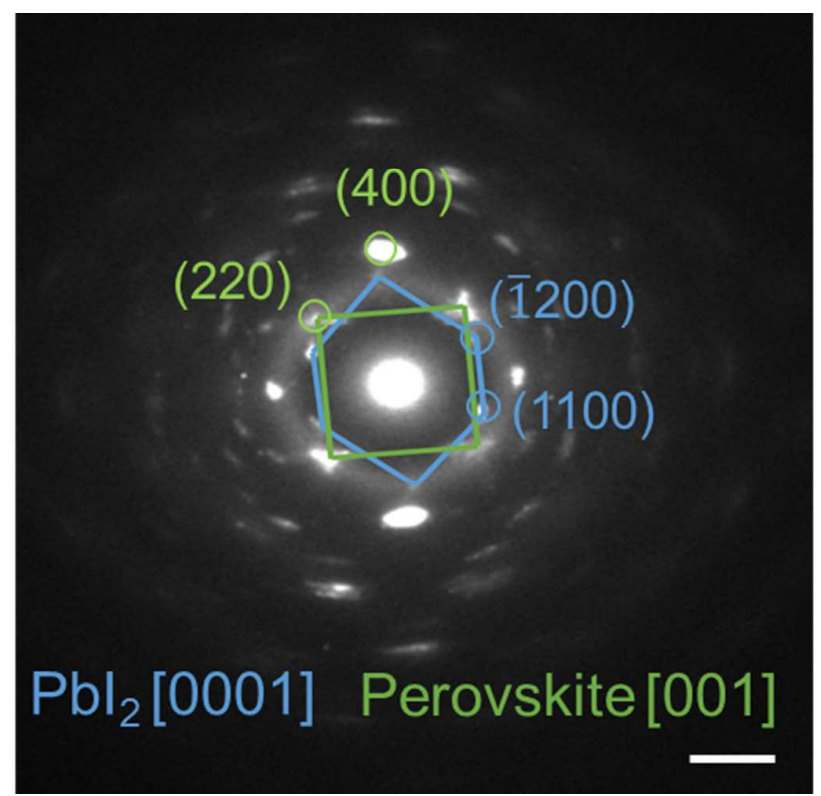

Figure S5 Electron diffraction pattern of 2D perovskite nanosheet under high energy electron beam irradiation. Scale bar: 2 1/nm.

It should be noted that the perovskite was sensitive to electron beam or laser irradiation, $\mathrm{PbI}_{2}$ reflections are also evident in the diffraction patterns under the long irradiation time (Figure S5). This is due to localized loss of $\mathrm{CH}_{3} \mathrm{NH}_{3} \mathrm{I}$ under electron beam or laser irradiation, leaving small regions of $\mathrm{PbI}_{2}$. The rewritable characteristic under electron beam or laser will make this type of perovskites promising candidates for fabricating novel functional devices, which needs further investigation. 
a

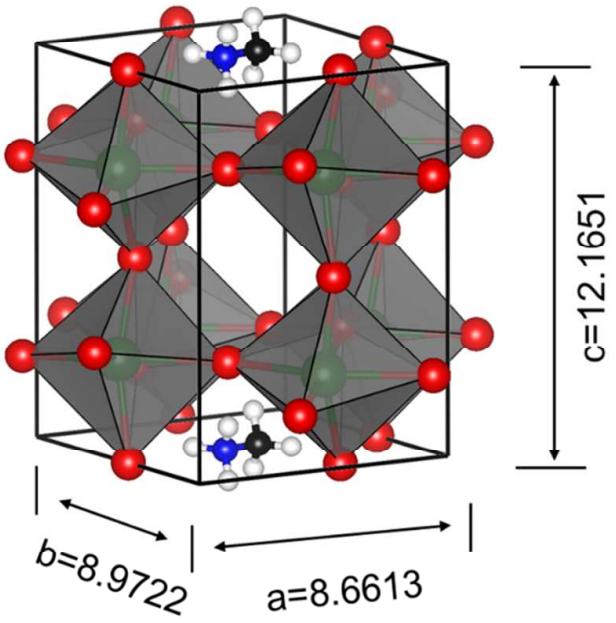

b

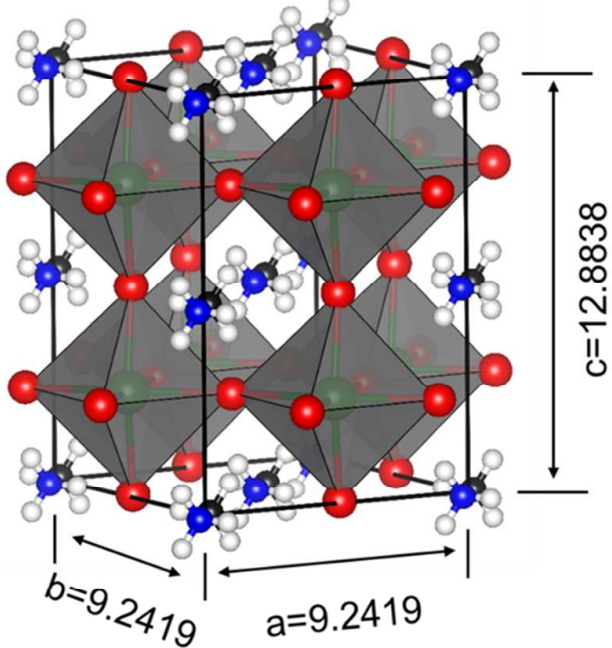

Figure S6 The relaxed structures for the tetragonal phase perovskite with different $\mathrm{MA}^{+}$intercalation ratios. (a) $\mathrm{CH}_{3} \mathrm{NH}_{3} / \mathrm{Pb}=0.25$. (b) $\mathrm{CH}_{3} \mathrm{NH}_{3} / \mathrm{Pb}=1$.

Table 1 The lattice constants of tetragonal perovskite $\left(\mathrm{AyBX}_{3}\right)$ with different intercalation ratios of $\mathrm{CH}_{3} \mathrm{NH}_{3}$.

\begin{tabular}{|l|l|l|}
\hline $\mathrm{y}$ & Lattice type & Lattice parameters, $a, b$ and $c$ in $\AA$ \\
\hline 0.25 & Monoclinic & $a=8.6613, b=8.9722, c=12.1651, \alpha=90.00^{\circ}, \beta=90.00^{\circ}, \gamma=86.43^{\circ}$ \\
\hline 0.5 & & $a=9.2047, b=8.9159, c=12.3357, \alpha=90.00^{\circ}, \beta=90.00^{\circ}, \gamma=86.53^{\circ}$ \\
\hline 0.75 & & $a=8.9251, b=8.9608, c=12.6323, \alpha=90.02^{\circ}, \beta=90.29^{\circ}, \gamma=89.35^{\circ}$ \\
\hline 1 & Tetragonal & $a=b=9.2419, c=12.8838, \alpha=\beta=\gamma=90.00^{\circ}$ \\
\hline
\end{tabular}

All the calculations were performed by Vienna Ab-initio Simulation Package (VASP). Project augment wave (PAW) method was used to describe the interaction between the ion and valence electrons. The exchange-correlation functional developed by Perdew, Burke and Ernzerhof (PBE) was used. The energy cutoff of all the calculations was set to $500 \mathrm{eV}$. A Monkhorst-pack k-point mesh with density of $4 \times 4 \times 2$ was used in the calculations of tetragonal phase. The tetragonal phase of $\mathrm{ABX}_{3}$ was modelled by a $\sqrt{2} \times \sqrt{2} \times 2$ supercell of the cubic unit cell with four $\mathrm{CH}_{3} \mathrm{NH}_{3}$ 
molecules in it. To simulate the perovskite crystal structure with different $\mathrm{CH}_{3} \mathrm{NH}_{3}$ molecules, the corresponding numbers of $\mathrm{CH}_{3} \mathrm{NH}_{3}$ molecules were added from the simulation cell. In the initial structure, the $\mathrm{C}-\mathrm{N}$ bond of all $\mathrm{CH}_{3} \mathrm{NH}_{3}$ molecules was set to align in the $<100>$ direction of the cubic unit cell. Then all the structures were relaxed until the force exerted on each atom is less than $0.01 \mathrm{eV} / \AA^{2}$.

The DFT calculations results (Figure S6) suggest that the crystal structure transforms from hexagonal to monoclinic system when the $\mathrm{CH}_{3} \mathrm{NH}_{3} \mathrm{I}$ molecules start to intercalate into the interval sites of $\mathrm{PbI}_{6}$ octahedrons layers. However, due to the completely conversion of $\mathrm{PbI}_{2}$ into $\mathrm{CH}_{3} \mathrm{NH}_{3} \mathrm{PbI}_{3}$ perovskite, the length of $c$-axis could be increased and the shear stress in $a-b$ plane will be introduced, which changes the $\gamma$ angle. As a result, the lattice changes from monoclinic to tetragonal structure.

a
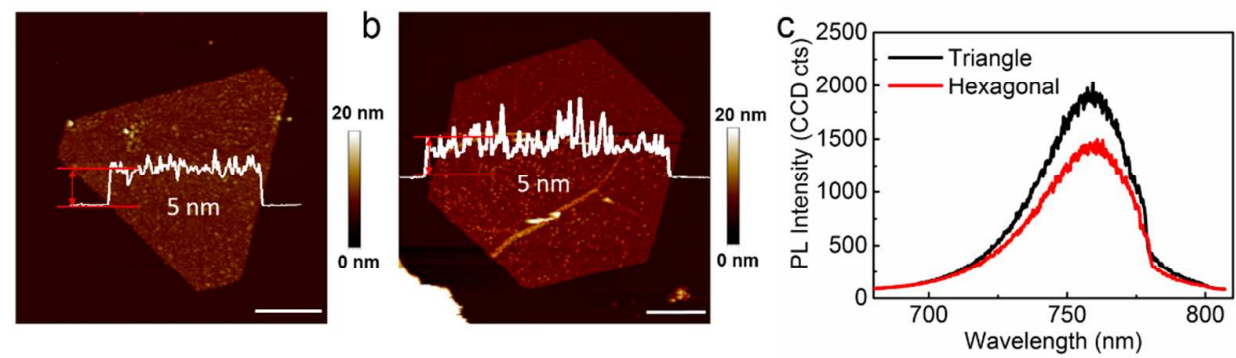

Figure $\mathrm{S} 7$ (a) and (b) AFM topography images of $2 \mathrm{D} \mathrm{CH}_{3} \mathrm{NH}_{3} \mathrm{PbI}_{3}$ nanosheets with the same thickness but different shapes (a: Triangle; b: Hexagon). Scale bars: $2 \mu \mathrm{m}$. (c) PL spectra collected from a and $b$.

Figure S7 a and b show the AFM topography image of the truncated triangular and hexagonal nanosheets with the same thickness. The PL spectra in Figure S7c are collected from the sample in a and b. It can be seen that these is no clear PL peak shift between these two spectra, indicating the same phase and structure. The intensity 
difference of the PL spectra might be due to the different laser intensities, focusing conditions or integration times.

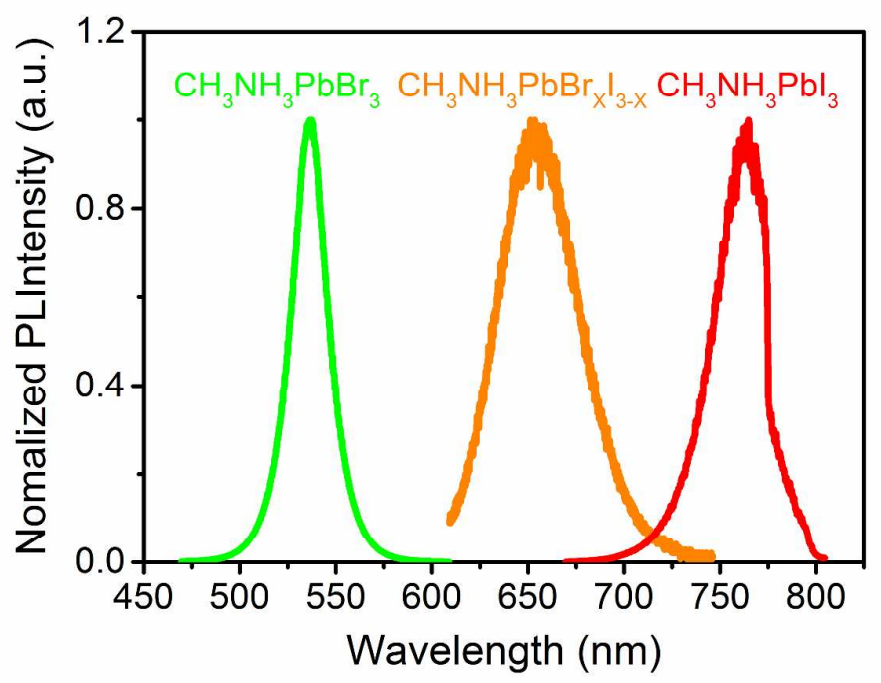

Figure S8 Nomalized PL spectra of 2D perovskite nanosheets with different compositions. Red trace: $\mathrm{CH}_{3} \mathrm{NH}_{3} \mathrm{PbI}_{3}$; orange trace: $\mathrm{CH}_{3} \mathrm{NH}_{3} \mathrm{PbBr}_{\mathrm{x}} \mathrm{I}_{3-\mathrm{x}}$; green trace: $\mathrm{CH}_{3} \mathrm{NH}_{3} \mathrm{PbBr}_{3}$. 


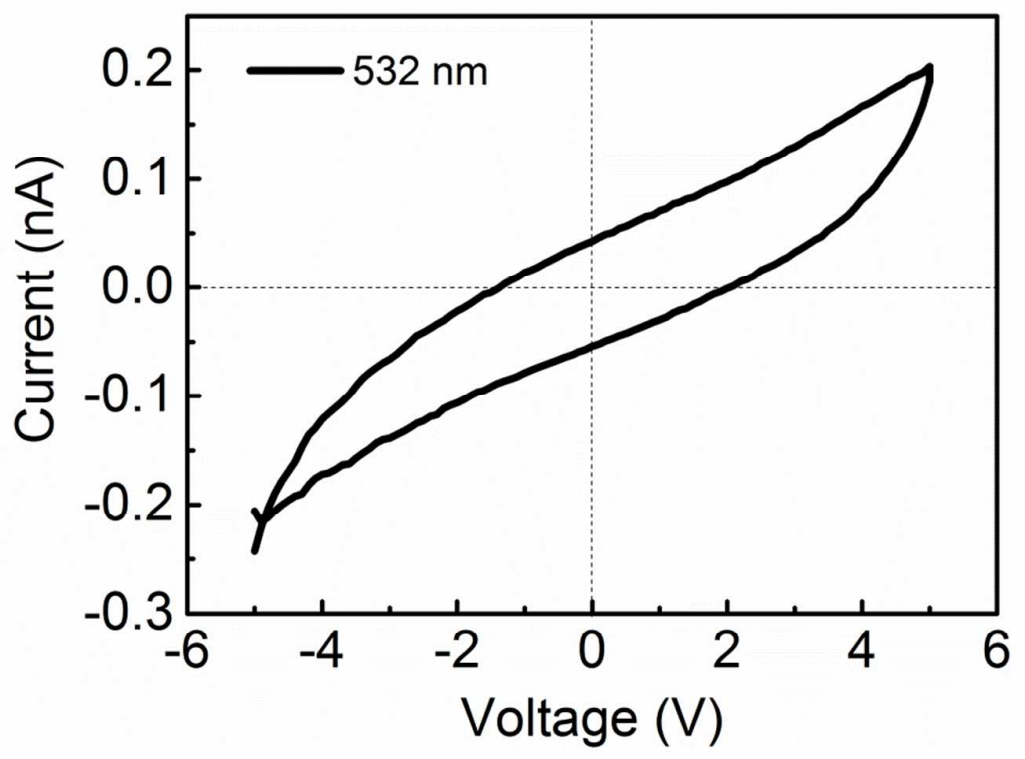

Figure S9 I-V curves of the 2D perovskite based device under the irradiation of weak unfocused $532 \mathrm{~nm}$ laser.

When the 2D perovskite based device was illuminated by weak laser (405 nm or $532 \mathrm{~nm}$, unfocused, the effective power: $<10 \mathrm{pw}$ ), the hysteresis can be observed in I-V curves, i.e. the current depends on the direction of the voltage sweeping between the source and drain electrodes. This means that some displacement (i.e. charge concentrations) related to the light intensity may happened, which could not be introduced by charge carriers injected from the contacts under forward bias. 

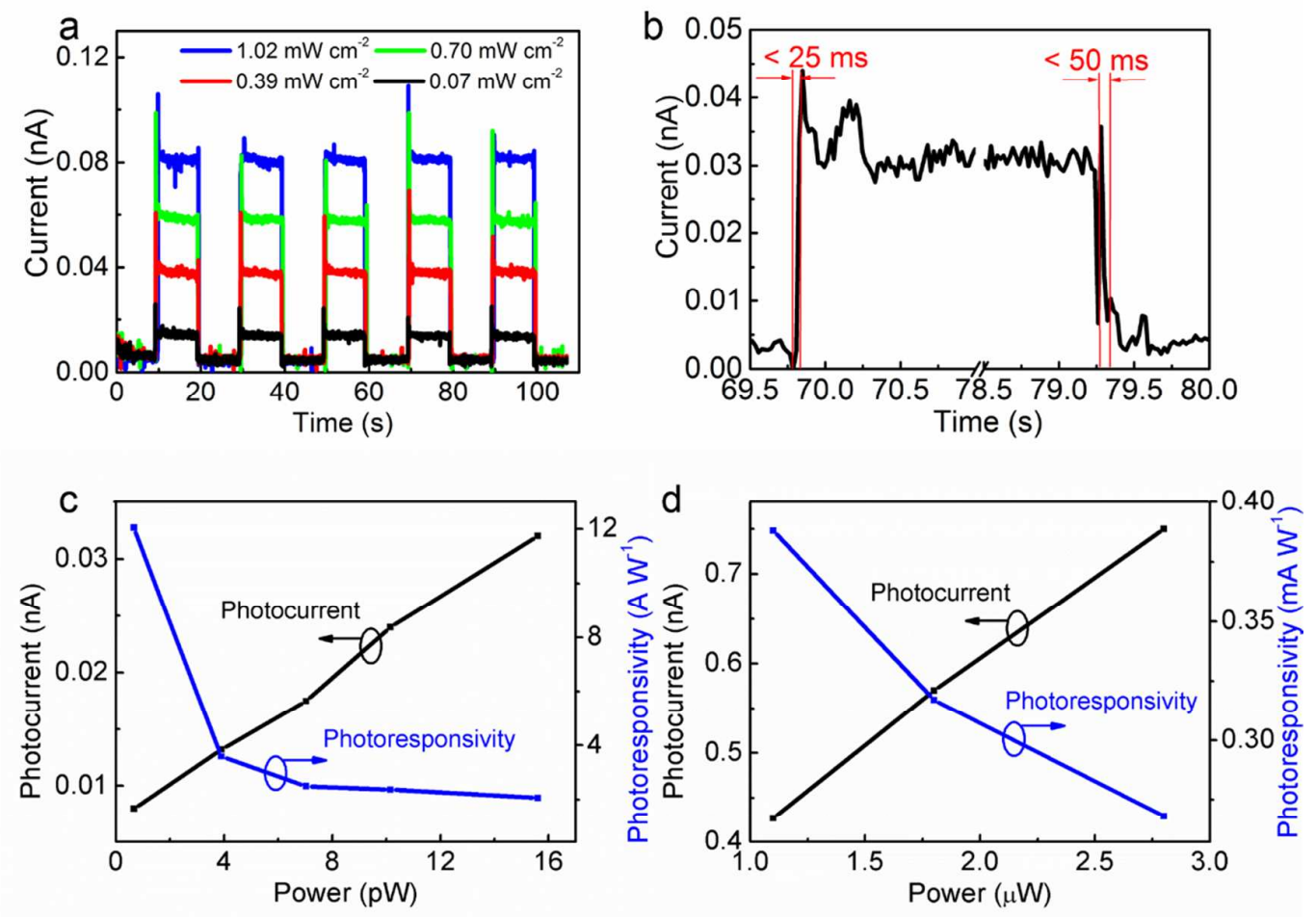

Figure S10 (a) Time-dependent photocurrent measurement on perovskite phototransistor over a five-period on- off operation under the different illumination of 532 laser. (b) Temporal photocurrent response excited at $532 \mathrm{~nm}$. The rise time (< $25 \mathrm{~ms}$ ) is defined as the time for the photocurrent increased to $70 \%$ of ON-state current. The fall time $(<50 \mathrm{~ms})$ is defined as the time for the photocurrent decreased by $70 \%$ of $\mathrm{ON}$-state current. (c) Photocurrent and photoresponsivity versus optical illumination power at a wavelength of $532 \mathrm{~nm}$ with a voltage bias of $1 \mathrm{~V}$. (d) Photocurrent and photoresponsivity versus optical illumination power under natural light with a voltage bias of $1 \mathrm{~V}$.

Figure S10a shows the photocurrent response of the device under the irradiation of the $532 \mathrm{~nm}$ laser with different power. Compared with the photocurrent response under the irradiation of the $405 \mathrm{~nm}$ laser, it can be found that the higher photocurrent was generated under $405 \mathrm{~nm}$ laser, in agree with the higher absorbance at a shorter wavelength. Temporal photocurrent response of the transistor under $532 \mathrm{~nm}$ laser was measured, as shown in figure $\mathrm{S} 9 \mathrm{~b}$. The result reveals that a rise time less than $25 \mathrm{~ms}$ and a fall time less than $50 \mathrm{~ms}(70 \%)$, which can be applicable for many applications. 
Figure S10c and d shows the dependence of photocurrent and photoresponsivity on $532 \mathrm{~nm}$ laser (c) and natural light (d) with a voltage bias of $1 \mathrm{~V}$. The photocurrent is increased linearly with promoting the light power. It can be seen that higher photoresponsivity can be achieved when decreasing the illumination power.

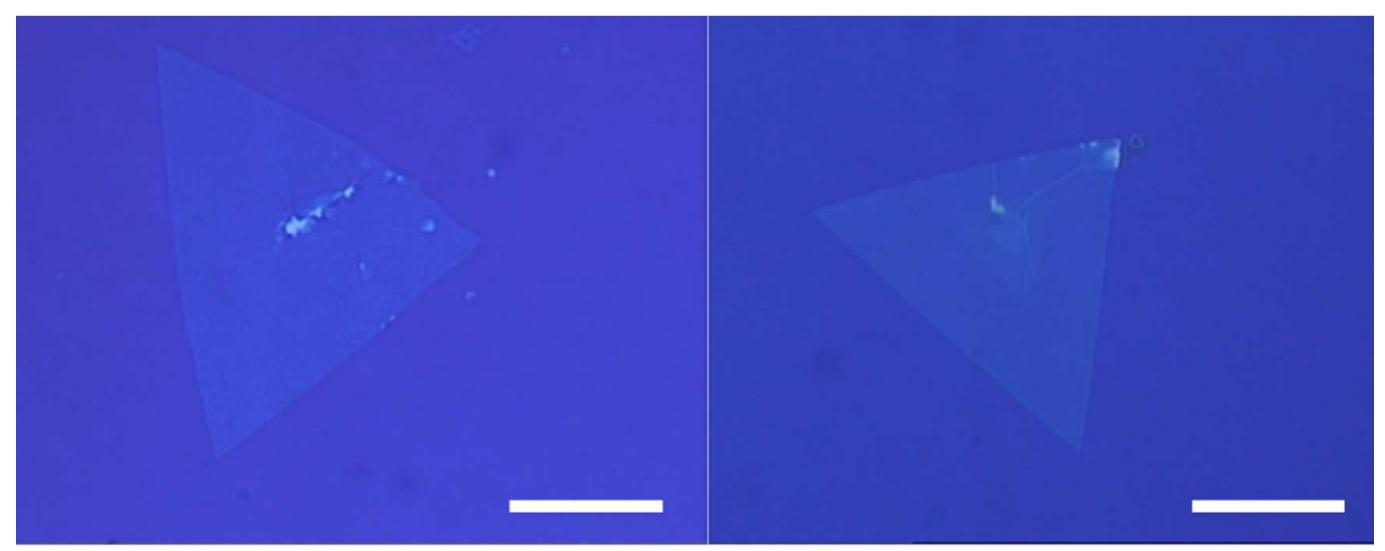

Figure S11. Optical images of larger size single crystal perovskite 2D nanosheets. Scale bars: $20 \mu \mathrm{m}$. 